\title{
Editorial
}

Manuelle Medizin 2012 · 50:257-259

DOI 10.1007/s00337-012-0935-2

๑) Springer-Verlag 2012

\author{
L. Beyer ${ }^{1} \cdot$ J. Meyer zu Tittingdorf ${ }^{2}$ \\ ${ }^{1}$ Geschäftsstelle DGMM, Ärztehaus Mitte, Jena \\ ${ }^{2}$ Redaktion, Springer-Verlag GmbH, Heidelberg
}

\section{Band 50 von Manuelle Medizin}

Liebe Leserinnen, liebe Leser,

mit Heft 1 wurde 2012 der Band Nr. 50 von Manuelle Medizin aufgeschlagen. Ein runder Geburtstag - Anlass für Herausgeber und Verlag, Rückschau auf den Weg unserer Zeitschrift zu halten.

Wir erinnern uns an die mutigen Kollegen, die in den ersten Jahren die Manuelle Medizin gegen den operativen Mainstream etablierten ( $\bullet$ Abb. 1). In diesen stürmischen Zeiten begleitete die Zeitschrift $\mathrm{Ma}$ nuelle Medizin den Aufbau eines Schulsystems sowie die Präsenz und Akzeptanz im ambulanten, stationären und rehabilitativen Versorgungsbereich. Die Manuelle Medizin wurde lehr- und lernbar gemacht.

\section{Jahre Begleiter von Praxis und Wissenschaft}

Durch die Jahrzehnte war die Zeitschrift - und ist es bis heute - Spiegel der gewünschten Vielfalt der Erfahrungen, Ansichten und Überlegungen aus manual- medizinischer Praxis und naturwissenschaftlichen Grundlagen (• Abb. 2).

Wie liest sich heute ein Beitrag, der vor 45 oder 30 Jahren veröffentlicht wurde? Reprintbeiträge aus den einzelnen Jahrzehnten sollen verdeutlichen, wie die Zeitschrift eine Basis für das Einbetten der Manualmedizin in die Fachgebiete der Schulmedizin wurde.

Gutmann, ein Kollege der ersten Stunde der Manuellen Medizin in Deutschland, schrieb 1968 über „das cervical-diencephal-statische Syndrom des Kleinkindes“. Von Heymann kommentiert: „Der hier nachgedruckte Artikel war der erste überhaupt, der die vielfältigen und typischerweise oft unpräzisen Symptome hirnstammvermittelter Reaktionen auf funktionelle Störungen der oberen Halswirbelsäule von Kleinkindern beschrieb. Wir sind froh, dass wir den Fortschritt der Erkenntnisse über die Manuelle Medizin bei Kindern, der mit diesem Artikel angestoßen wurde, über die 50 Jahre der Zeitschrift Manuelle Medizin verfolgen können.“
Hausammann, ein Schweizer Chirurg, fasste 1971 die Funktionsstörungen in einem Artikel „Hüftschmerz und Iliosakralgelenk“ zusammen. Die Diskrepanz zwischen Röntgenbild und Beschwerdebild, bekannt von der Wirbelsäule, wurde auch am Hüftschmerz sichtbar. W. Beyer kommentiert diesen Beitrag und stellt fest, dass hier „der Grundgedanke des projizierten Schmerzes und des daraus resultierenden Denkens in Ketten dargelegt wurde, der das zentrale Element im manualmedizinischen Denken und Handeln“ geworden ist. „Kasuistik und sorgfältige klinische Beobachtung waren typisch zur damaligen Zeit, genügen aber heutigen wissenschaftlichen Ansprüchen nicht mehr.“

Interessant war es festzustellen, dass die Zusammenfassung der Beiträge in die englische, französische und russische Sprache übersetzt wurden.

Mattle, ein Neurologe aus Bern, schrieb 1987 über „das gefährliche Zervikalsyndrom“. „Es sind Affektionen fast aller

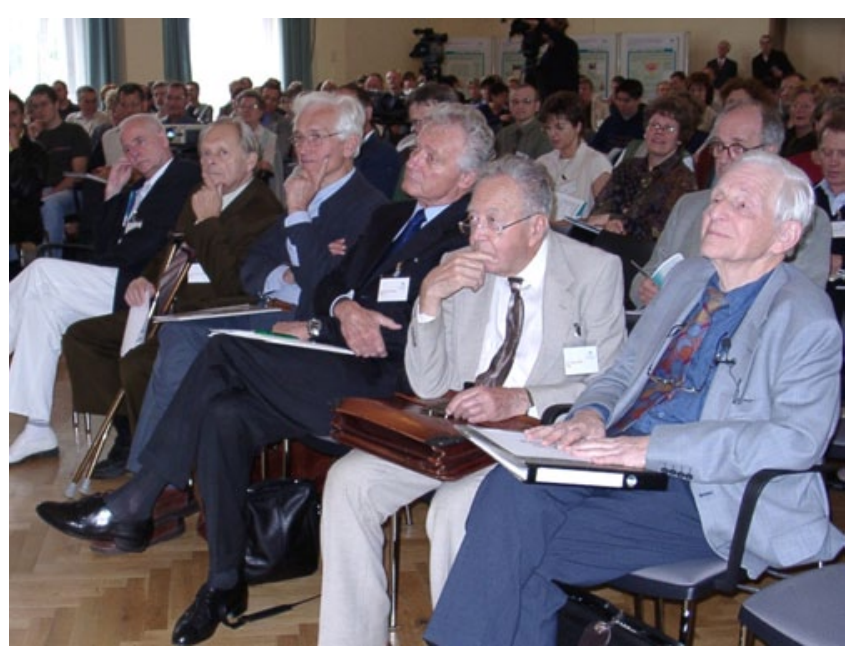

Abb. $1 \Delta$ Dr. Wolff, Prof. Lewit, Prof. Tilscher, Prof. Conradi, Prof. Janda (v.r.n.l.) bei einem Kongress in der Klinik Sommerfeld bei Berlin. Mit freundl. Genehmigung von Dr. v. Heymann

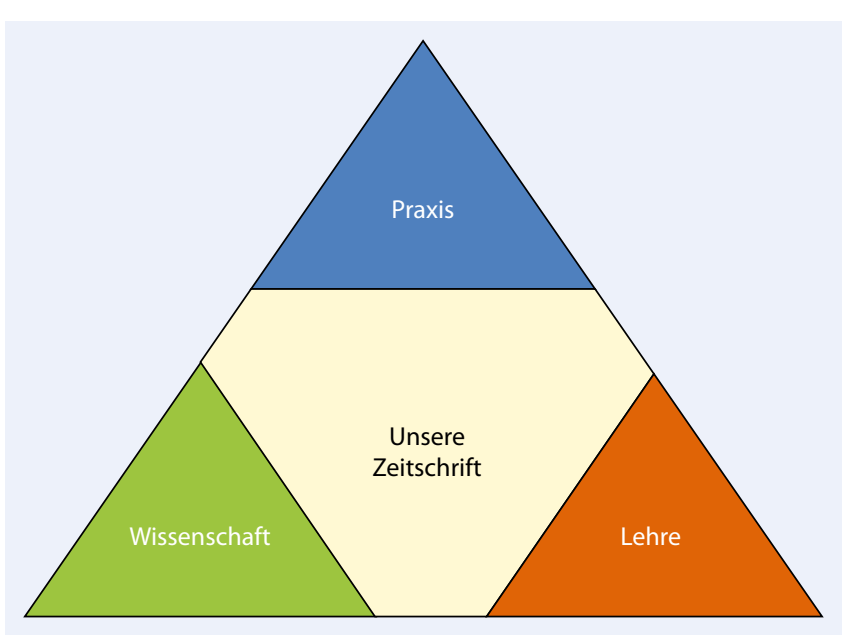

Abb. $2 \Delta$ Die Zeitschrift als Schnittmenge und Klammer 


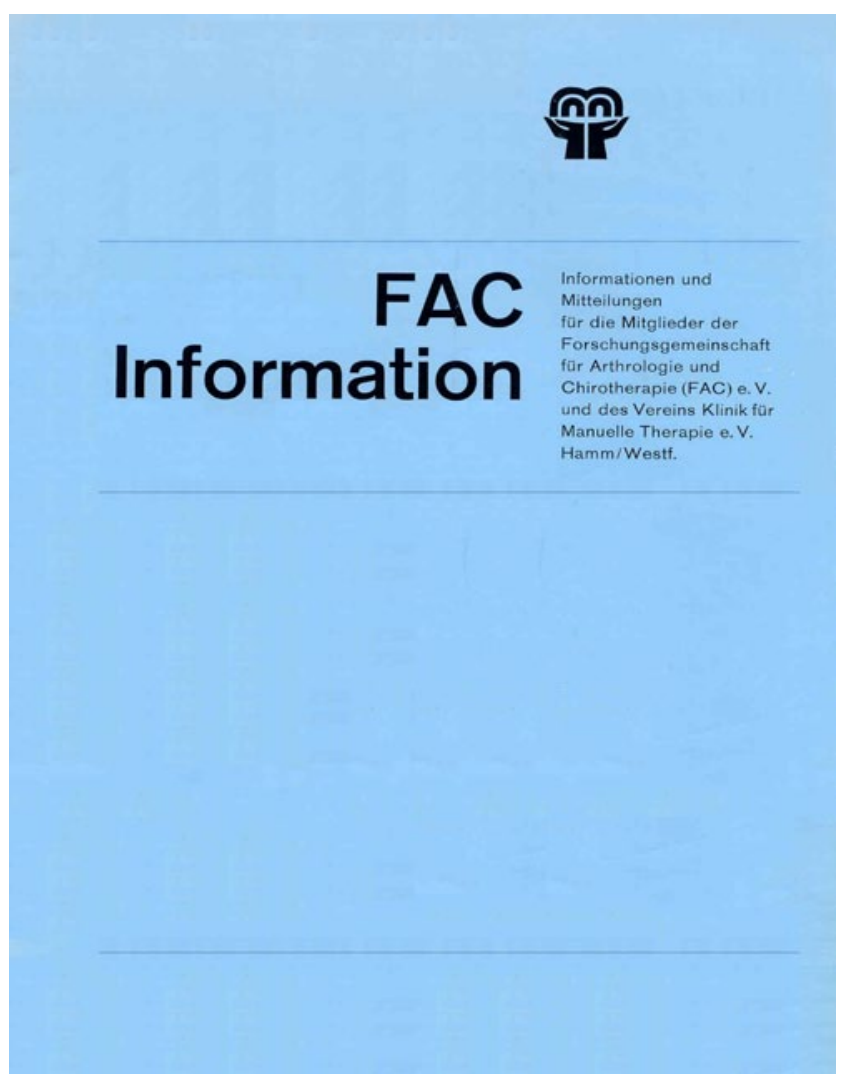

Abb. $3<$ Cover der ersten Ausgabe aus dem Jahr 1963, damals noch unter dem Namen FAC-Information.

Strukturen in der Wirbelsäule geeignet, infolge reflektorischer Schmerzmechanismen ein Zervikalsyndrom auszulösen.“ Lackner kommentiert diesen Beitrag unter der Überschrift „Die Bedeutung organdiagnostischer Darstellungen für die manuelle Medizin“ und stellt dar, wie die Diskussion in unserer Zeitschrift dazu beigetragen hat, eine differenzierte, funktionelle Betrachtungsweise funktionell bedingter Beschwerden zu entwickeln. Im Kommentar wird auch deutlich, dass die Zeitschrift unverzichtbares Mittel ist für den fruchtbaren Streit um Inhalte, Terminologie und Definitionen, wie er in jedem wissenschaftlichen Fachbereich geführt werden muss.

Weitere Reprintbeiträge sind:

- Tilscher, Hanna, Graf (1994) „Klinische und röntgenologische Befunde bei der Hypermobilität und Instabilität im Lendenwirbelsäulenbereich“

- Sachse (1998) „Differentialdiagnostik der reversiblen hypomobilen Dysfunktion", kommentiert von Harke und Linz

- von Heymann (2005) „Grundlagenforschung trifft Manualmedizin“
In „Grußworten“ beschreiben die manualmedizinischen Gesellschaften im deutschsprachigen Raum die Bedeutung einer manualmedizinischen Zeitschrift für die Mitglieder ihrer Gesellschaften. Die Zeitschrift gewährt Rückblick auf die eigene Entwicklung der Seminare. „Dass sich die abgespaltene osteopathische Gesellschaft der Weiterentwicklung durch Diskussion mit den manualmedizinischen Kollegen im bewährten Rahmen von Manuelle Medizin entzogen hat und in der eigenen Zeitschrift mit wissenschaftlich teils zweifelhaften Partnern in einem Boot sitzt, hat der Osteopathie sicher nicht genützt“, wird hier resümiert.

\section{Eine Zeitschrift im Wandel}

Aber nicht nur das Fach Manuelle Medizin blickt auf eine spannende Entwicklung zurück. Auch die Zeitschrift selbst hat vielfach Veränderungen durchlaufen. Mal waren diese personeller Natur, mal wurden neue inhaltliche Schwerpunkte definiert und hin und wieder war ein Facelifting nötig, um die Zeitschrift dem Geschmack der Zeit anzupassen.
Personelle Veränderungen gab es selbstverständlich auch aufseiten des Verlags, doch wirklich bedeutend für die Entwicklung der Zeitschrift waren die Köpfe, die als Schriftleiter und Herausgeber die Zeitschrift in ihrer Ausrichtung und ihrem Inhalt über die Jahrzehnte entscheidend geprägt haben. Einigen von ihnen haben wir in dieser Jubiläumsausgabe Raum gewidmet, in dem Kollegen ganz individuell Danke sagen.

Als Spiegel für die konzeptionelle Weiterentwicklung können die Rubriken und der Untertitel der Zeitschrift gelten. Konstanten wie Übersichten, Originalien und Kasuistiken charakterisieren die Zeitschrift seit ihren Anfängen bis heute, andere Rubriken sind dagegen im Laufe der Jahre neu hinzugekommen. Waren ehemals Buchbesprechungen ein wiederkehrendes Element in jeder Ausgabe, so wuchs über die Jahre stetig die Bedeutung internationaler Fachzeitschriften auch für den deutschsprachigen Manualmediziner und Physiotherapeuten. Dementsprechend folgerichtig wurde 2006 die Rubrik „Literatur im Fokus“ eingeführt. Ebenfalls konsequent war die Einführung der Rubrik „CME“ im Jahr 2011, die es im Rahmen der Springer e.Akademie (www.springermedizin.de/eAkademie) ermöglicht, die gesetzlich verlangten Fortbildungspunkte mit manualmedizinisch spezifischen Beiträgen zumindest teilweise zu erlangen. Nur wenigen dürfte aber bekannt sein, dass gerade im Bereich Fortbildung die Manuelle Medizin ihrer Zeit bereits einmal weit voraus war. Schon Anfang der 1990er Jahre gab es eine Rubrik „Fortbildung“, die eigens erstellte Übersichten enthielt und zum Ziel hatte, den State of the Art zu einzelnen Techniken und Verfahren übersichtlich abzubilden. Um diese schon damals sehr aufwendige Rubrik regelmäßig erscheinen zu lassen, bedurfte es jedoch großer Anstrengungen und eines langen Atems. Dieser reichte nicht ganz bis in das Jahr 2004, bis die Fortbildung schließlich vom Gesetzgeber verpflichtend geregelt wurde.

Auch der Untertitel der Zeitschrift durchlebte bis heute zwei Veränderungen. Reichte anfänglich noch der Titel Manuelle Medizin aus, so wurde dieser später ergänzt durch den Untertitel Chirotherapie - Manuelle Therapie - Osteopathische Me- 


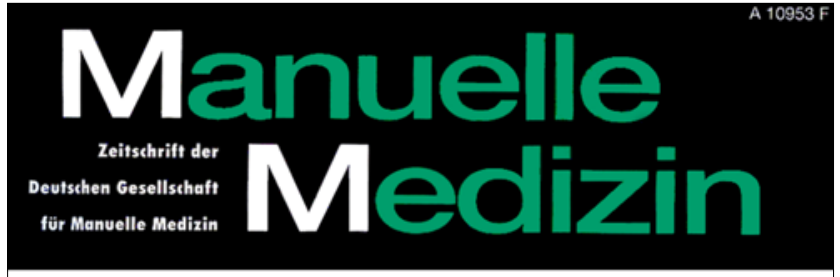

33. Jahrgang

Eebruar 1995

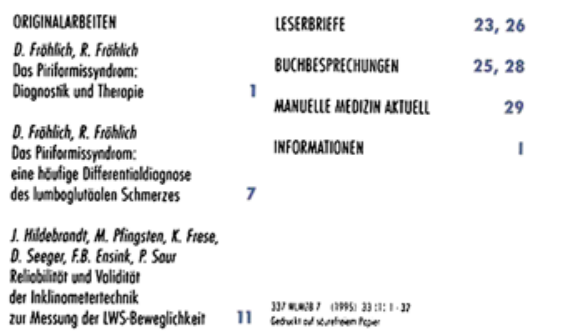

Springer

Factors die Manuelle Medizin international besser sichtbar und somit noch attraktiver für Autoren zu gestalten.

Was hält die Zukunft für die Manuelle Medizin noch bereit? Wird es in 10 Jahren Usus sein, dass Sie Ihre Zeitschrift auf einem Tablet-PC lesen? Erwarten uns nur noch kurze Beiträge, die umfangreiche Videosequenzen flankieren, oder aber werden Sie noch immer in bewährter Weise durch Ihre Zeitschrift blättern? Wir dürfen gespannt sein. Für den Augenblick sei es aber allen Beteiligten gegönnt, mit ein wenig Stolz und Zufriedenheit auf die ersten, erfolgreichen 50 Jahrgänge von Manuelle Medizin zu blicken.

Ihnen, verehrte Leserinnen und Leser, wünschen wir an dieser Stelle viel Vergnügen, wenn Sie uns auf einer kleinen Zeitreise durch die Manuelle Medizin begleiten.

Abb. $4<$ Cover einer Ausgabe aus dem Jahr 1995. Mit freundl. Genehmigung von Dr. v. Heymann dizin, ein Zeichen für die thematisch breite Aufstellung der Zeitschrift. 2005 erfolgte dann eine bewusste Fokussierung und der dritte Bestandteil verschwand nahezu unbemerkt vom Cover.

Wie sich das Layout von Manuelle Medizin verändert hat, zeigen die Beiträge aus den vergangenen Jahrzehnten, die wir in dieser Ausgabe ganz bewusst im „historischen“ Layout belassen haben. Schon beim bloßen Durchblättern werden Sie erahnen können, aus welchem Jahrzehnt der jeweilige Beitrag stammt. Und auch ein Blick auf das Cover der ersten Ausgabe im Jahr 1963 (• Abb. 3) sowie einer Ausgabe aus dem Jahr 1995 (• Abb. 4) wird einige darüber schmunzeln lassen, mit wie wenig Farbe und gestalterischen Mitteln früher Zeitschriften „gestaltet" wurden. Manch einer unter Ihnen mag sich beim Anblick der alten Artikel und Cover aber auch etwas wehmütig an die „guten alten Zeiten" erinnern.

Und genau diese guten alten Zeiten wollen Herausgeber und Verlag in den kommenden Monaten tatsächlich zurückholen. Die „historische“ Beiträge in dieser Jubiläumsausgabe belegen eindrucks- voll, welche Schätze sich auch in den weiter zurückliegenden Jahrgängen von Manuelle Medizin finden. Bis dato sind jedoch nur die Jahrgänge ab 1997 über das Onlinearchiv auf www.ManuelleMedizin. springer.de verfügbar. Um hier Abhilfe zu schaffen, werden aktuell alle Ausgaben, die bisher nur gedruckt vorlagen, digitalisiert. Ziel ist es, schließlich auf alle Beiträge, die ab der ersten Ausgabe von Manuelle Medizin erschienen sind, komfortabel online zugreifen zu können.

Dies ist nur eins der Projekte, die die Manuelle Medizin zukunftsfähig machen sollen. Ein weiteres Ziel ist die Öffnung der Zeitschrift über die deutschen Sprachgrenzen hinaus für Manualmediziner aus ganz Europa, denn nicht nur in Deutschland, Österreich und in der Schweiz wird hervorragende Manuelle Medizin praktiziert. Dies wäre zudem ein wichtiger Schritt in Richtung eines Impact Factors für die Manuelle Medizin. Man mag über Sinn und Unsinn des Impact Factors trefflich streiten, doch ist seine Bedeutung ganz besonders für junge Mediziner unbestritten. Es muss daher ein Anliegen aller sein, durch den Erhalt eines Impact
Ihre

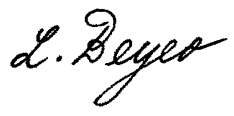

L. Beyer

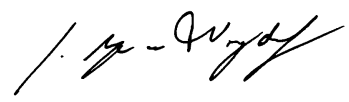

J. Meyer zu Tittingdorf

\section{Korrespondenzadresse}

\section{Prof. Dr. L. Beyer}

Geschäftsstelle DGMM, Ärztehaus Mitte

Westbahnhofstr. 2, 07745 Jena

lobeyer@t-online.de 